\title{
FACTORES DEL ESTILO DE VIDA PREDISPONENTES A DISPLASIA CERVICAL EN MUJERES ATENDIDAS EN EL HOSPITAL MARÍA AUXILIADORA, 2013-2017
}

\author{
LIFESTYLE FACTORS PREDISPONENT TO CERVICAL DYSPLASIA IN WOMEN ATTENDED IN THE \\ HOSPITAL MARÍA AUXILIADORA, 2013-2017
}

Rocio Gallegos Toribio', Milagros A. Fuentes Vargas³,b, Magdiel José Manuel Gonzales Menéndezª,c

\begin{abstract}
RESUMEN
Introduccion: La displasia cervical es la lesión precursora del cáncer de cuello uterino, en el que se observan alteraciones de las células cervicales sin llegar a adoptar características de malignidad. Objetivo: Identificar los factores predisponentes para displasia cervical en las pacientes atendidas en el hospital María Auxiliadora durante el 2013 al 2017. Métodos: Estudio de diseño casos y controles, analítico y observacional. Se analizaron 216 historias clínicas de pacientes, dividiéndose en 72 casos y 144 controles. Resultados: En el análisis bivariado, resultaron significativos la menarquia temprana $(O R=2,071 ; p=0.024)$, el inicio temprano de relaciones sexuales $(O R=2,213 ; p=0.017)$, el tener múltiples parejas sexuales $(O R=3,036 ; p=0.001)$ y la multiparidad $(O R=2,316 ; p=0.005)$. En el análisis multivariado las variables independientes fueron el tener múltiples parejas sexuales $(O R=2,626 ; p=0.003)$ y la multiparidad $(\mathrm{OR}=2,759 ; \mathrm{p}=0.045)$ las cuales resultaron significativas. Conclusión: Se concluye que los factores del estilo de vida predisponentes a displasia cervical fueron tener múltiples parejas sexuales y la multiparidad.

Palabras clave: Displasia cervical; Número de parejas sexuales; Multiparidad; Factores predisponentes; Medicina preventiva; Estilos de vida. (fuente: DeCS BIREME)
\end{abstract}

\begin{abstract}
Introduction: The cervical clearance is the precursor lesion of cancer of the cervix, in which alterations of the cervical cells are observed. Objective: To identify the predisposing factors for the cervical dispute in the patients treated at the María Auxiliadora Hospital during 2013 to 2017. Methods: Design study, cases, controls, analytical and observational. 216 clinical records of patients will be analyzed, divided into 72 cases and 144 controls. Results: In the bivariate analysis, significant results in early menarche $(O R=2.071, p=$ $0.024)$, early onset of sexual relations $(O R=2.213, p=0.017)$, having multiple sexual partners $(O R=3.036 ;=$ $0.001)$ and multiparity $(O R=2.316, p=0.005)$. In the multivariate analysis, the independent variables were multiple sexual partners $(O R=2.626, p=0.003)$ and multiparity $(O R=2.759, p=0.045)$. Conclusion: It is concluded that lifestyle factors predispose a cervical dispute.
\end{abstract}

Key words: Cervical dysplasia; Number of sexual partners; Multiparity; Predisposing factors; Preventive medicine; Lifestyles. (source: MeSH NLM)

\footnotetext{
Hospital María Auxiliadora, Lima-Perú.

2 Facultad de Medicina Humana, UCSM, Arequipa-Perú.

${ }^{3}$ Instituto de Investigación en Ciencias Biomédicas, Universidad Ricardo Palma, Lima-Perú.

Médico Cirujano.

Estudiante de Medicina.

Especialista en Cirugía General.
}

Citar como: Rocio Gallegos Toribio, Milagros A. Fuentes Vargas, Magdiel José Manuel Gonzales Menéndez. Factores del estilo de vida predisponentes a displasia cervical en mujeres atendidas en el Hospital María Auxiliadora, 2013-2017. [Artículo Original].2019;19(2):48-56. (Abril 2019). DOI 10.25176/RFMH.v19.n2.2065

(c) Los autores. Este artículo es publicado por la Revista de la Facultad de Medicina Humana, Universidad Ricardo Palma. Este es un artículo de Open Access distribuido bajo los términos de la Licencia Creative Com- mons: Creative Commons Attribution 4.0 International(CC BY 4.0). (https://creativecommons.org/licenses/by/4.0/), que permite el uso no comercial, distribución y reproducción en cualquier medio, siempre que la obra original sea debidamente citadas. Para uso comercial, por favor póngase en contacto con revista.medicina@urp.pe 


\section{INTRODUCCIÓN}

El cáncer cervicouterino (CCU) se define como aquella neoplasia maligna que afecta a las células que revisten el cuello uterino. La displasia cervical es la lesión precursora del cáncer de cuello uterino, en el que se observan alteraciones de las células cervicales sin llegar a adoptar características de malignidad'.

De acuerdo a la Organización Mundial de la Salud (OMS), es el cuarto cáncer más frecuente en la mujer. Se calcula que en 2018 hubo 570000 nuevos casos, que representaron el 7,5\% de la mortalidad femenina por cáncer. De las aproximadamente 311000 defunciones por CCU que se registran cada año, más del $85 \%$ se producen en las regiones menos desarrolladas ${ }^{2}$.

Según GLOBOCAN, en el 2018 las mayores incidencias mundiales se presentan en las regiones de Sudáfrica y Sudamérica, con tasas mayores a 26,0 y 18,1 respectivamente, por cada 100000 habitantes. Sin embargo, EEUU, Canadá y Australia presentan las más bajas incidencias, presentando tasas menores a 7,3. Los países latinoamericanos con tasas de incidencia mayores de $30 \times 100000$ mujeres son Guyana $(44,7)$, Nicaragua $(39,9)$, Honduras $(37,8)$, El Salvador $(37,2)$, Bolivia $(36,4)$, Paraguay $(35,0)$, Perú $(34,5)$, Venezuela $(31,4)$ y Guatemala $(30,5)$. En el reporte de GLOBOCAN del 2018, el país latinoamericano con mayor incidencia de esta enfermedad es Bolivia, con una tasa de $38,5^{3}$.

En el Perú, cada 5 horas muere una mujer por cáncer cervical ${ }^{3}$. Según el perfil oncológico de los países publicado por la OMS en el 2014, en el Perú, el CCU es el cáncer más frecuente y a su vez, es el segundo cáncer con mayor mortalidad en la población femenina $(12,3$ por cada 100000 habitantes) ${ }^{4}$.Las regiones con tasas ajustadas de mortalidad por cáncer de cuello uterino más altas son Loreto $(18,0)$, Huánuco $(12,8)$, Ucayali $(10,3)$, con valores que duplican y hasta cuadruplican el de Lima $(4,2)^{5}$.

En el año 2015, por el servicio de Consultorio de Ginecología Oncológica del Hospital María Auxiliadora fueron atendidas 6627 pacientes, de las cuales se realizaron el 59\% (3965) estudios de tamizaje de citología cervical, haciéndose el diagnóstico de displasia cervical al $44.98 \%$ de mujeres (2981) lo que nos indica la importancia del estudio de esta lesión precancerosa para evitar su progresión a cáncer cervico uterino ${ }^{6}$.

El cuello uterino, consta de 2 partes: el ectocérvix, que está recubierto por un epitelio escamoso estratificado y el endocérvix cubierto por una única capa de epitelio cilíndrico. La metaplasia escamosa del cuello uterino consiste en el reemplazo fisiológico del epitelio cilíndrico evertido al exocérvix por un epitelio escamoso neoformado.El epitelio metaplásico puede evolucionar de dos modos. En la gran mayoría de mujeres, se convierte en epitelio metaplásico escamoso maduro, sin embargo, en una minoría puede evolucionar a epitelio atípico displásico. El CCU invasor de células escamosas viene precedidos por una larga fase de enfermedades preinvasoras, denominadas neoplasia intraepitelial cervical (NIC). Las NIC se clasifican en grados 1, 2 y 3 según la proporción del espesor epitelial que presenta células maduras y diferenciadas. La mayoría de las anomalías cervicales tienen escasa probabilidad de progresar a NIC o a cáncer cervicouterino de alto grado. La NIC de más bajo grado vuelve a la normalidad en plazos relativamente cortos o no progresa a alto grado. Es mucho más probable que la NIC de alto grado se convierta en cáncer invasor. La lesión precursora originada en el epitelio cilíndrico se denomina adenocarcinoma in situ (AIS). El AIS puede asociarse a NIC entre uno y dos tercios de los $\operatorname{casos}^{30}$.

El virus del papiloma humano (VPH) es una causa necesaria, pero no suficiente, para desarrollar cáncer de cuello uterino ${ }^{7,8}$. Los tipos de VPH 16 y 18 son responsables del $70 \%$ de cánceres cervicales 9 . Debido a que sólo una pequeña proporción de las infecciones de VPH progresan a cáncer, otros cofactores, externos y propios del huésped, deben estar involucrados en el proceso de carcinogénesis.

Por esta razón, se realizó el presente estudio con el fin de determinar los factores predisponentes al desarrollo de displasia cervical.

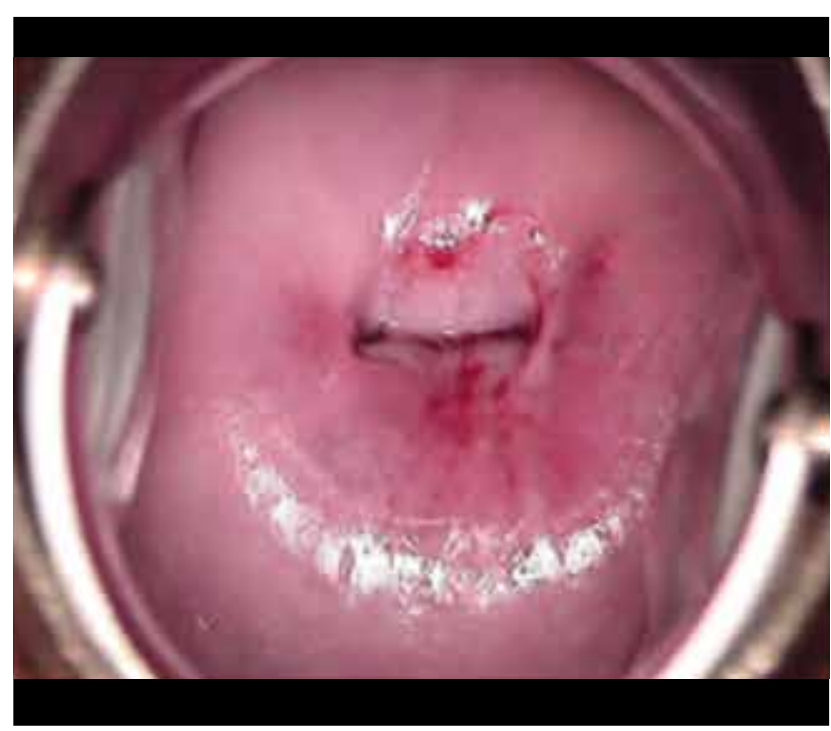




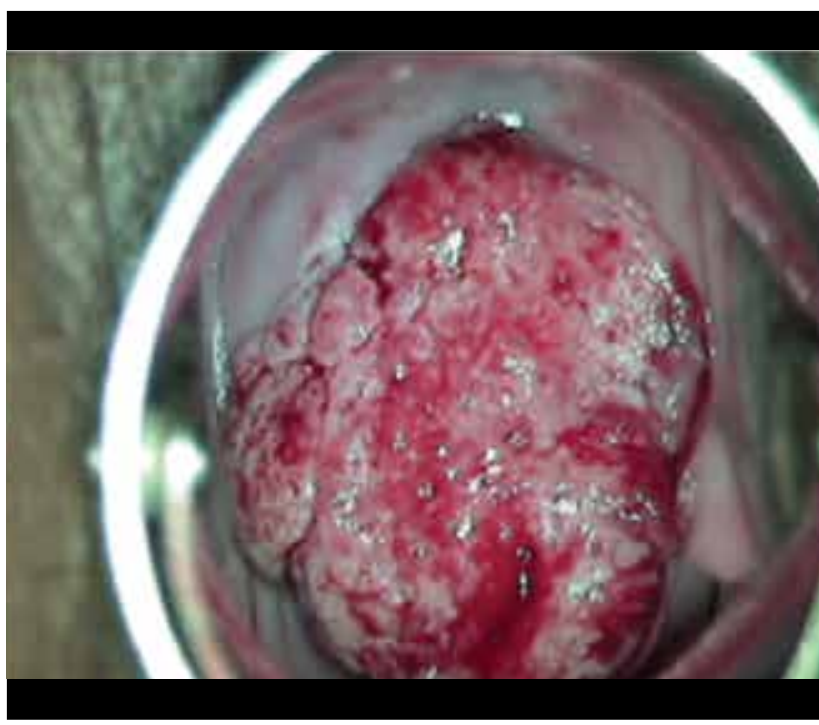

Figura 1. Vista de Displasia cervical de bajo grado (NIC I) y cáncer invasor.

\section{MÉTODOS}

Estudio de diseño casos y controles, observacional, retrospectivo y analítico. La población estuvo conformada por todas las pacientes que tuvieron estudio de citología cervical, con o sin diagnóstico de displasia cervical en el Servicio de Ginecología Oncológica del Hospital María Auxiliadora durante el período 2013 al 2017. De acuerdo al estudio realizado por Ruiz et al, se tomó como referencia la prevalencia de $20 \%$ de citología cervical alterada en los controles. Por lo tanto, se estimó que la prevalencia en los casos fue del $38 \%$. Se consideró la posibilidad de obtener un OR de 2.5 , con un intervalo de confianza del $95 \%$ y un poder estadístico del $80 \%$, emparejando a las unidades de estudio en 2 controles por cada caso. La muestra fue de 216 pacientes: 72 casos donde se encuentran las pacientes con diagnóstico de displasia cervical; $y$ 144 controles, quienes no presentan diagnóstico de displasia cervical.

Los criterios de Inclusión fueron pacientes de sexo femenino con citología cervical alterada, con o sin diagnóstico de displasia cervical e historias clínicas con datos a estudiar completos.

Los criterios de exclusión fueron historias clínicas incompletas $y / 0$ ilegibles, pacientes gestantes, pacientes con diagnóstico de carcinoma invasor (cérvix) u otras neoplasias.

Las variables estudiadas fueron: displasia cervical, características sociodemográficas y características gineco-obstétricas.

La recolección de datos se realizó utilizando la base de datos de historias clínicas otorgada por la oficina de Estadística e Informática del Hospital María Auxiliadora. El análisis de los datos se realizó a partir de la base de datos construida en el programa estadístico SPSS versión 25 . Todas las comparaciones se realizaron con un intervalo de confianza de $95 \%$ y un grado de error de $0.05 \%(p<0.05)$.

Así mismo, el estudio fue aprobado por la oficina de apoyo a la docencia e investigación del Hospital María Auxiliadora.

\section{RESULTADOS}

En la tabla 1 se observó que, de acuerdo a las características sociodemográficas, el grado de instrucción predominante fue secundaria (70,8\%), siendo la única característica estadísticamente significativa $(p=0.016)$.

Así mismo la tabla 2 indica que la mayoría de los casos $(70,8 \%)$ presentó múltiples parejas sexuales, mayor a dos gestaciones (76.4\%) y mayor a dos partos (68.1\%).

La tabla 3, describe el análisis bivariado de las características estudiadas. La menarquia temprana incrementa el riesgo de presentar displasia cervical en 2,07 veces ( $P=0.024)$ y el inicio temprano de vida sexual aumenta la probabilidad de presentar esta enfermedad en 2,21 veces $(P=0.017)$. Similarmente, tener 3 o más parejas sexuales incrementa hasta 3,03 veces $(\mathrm{P}=0.001)$ el riesgo de padecer esta patología. Por otro lado, presentar más de 2 gestaciones aumentaría el riesgo de displasia en 1,77 veces, sin embargo, no es estadísticamente significativo $(P=0.078)$. No obstante, presentar 3 o más partos eleva la posibilidad de esta enfermedad hasta 2,31 veces $(\mathrm{P}=0.005)$. Por último, respecto al uso de métodos anticonceptivos, se trataría como un factor protector, pero dicho hallazgo no es estadísticamente significativo $(\mathrm{OR}=0,673 ; \mathrm{P}=0.173)$.

Así mismo, en la tabla 4, se identificó que el tener múltiples parejas sexuales $(O R=2,626 ; \mathrm{P}=0.003)$ y presentar más de 2 partos $(\mathrm{OR}=2,759 ; \mathrm{P}=0.045)$ son estadísticamente significativos para el desarrollo de displasia cervical.

Cabe mencionar que la presencia de infección por VIH no fue considerado como factor predisponente en el presente estudio, sin embargo se encontró que el $10,6 \%(n=23)$ de las pacientes tenían infección por este virus, de las cuales 14 pertenecían a los casos y las 9 restantes eran controles. 
Tabla 1. Características sociodemográficas de las pacientes con citología cervical anormal atendidas en el Hospital María Auxiliadora.

\begin{tabular}{|c|c|c|c|c|}
\hline & $\begin{array}{l}\text { CASOS } \\
(n=72)\end{array}$ & $\begin{array}{c}\text { CONTROLES } \\
(n=144)\end{array}$ & TOTAL & VALOR P \\
\hline Edad (\%) & & & & 0.066 \\
\hline Menor a 31 años & $7(9.7)$ & $27(18.1)$ & $34(15.3)$ & \\
\hline 31-50 años & $45(62.5)$ & $93(64.6)$ & $138(63.9)$ & \\
\hline Mayor a 50 años & $20(27.8)$ & $24(16.7)$ & $44(20.4)$ & \\
\hline Estado civil (\%) & & & & 0.828 \\
\hline Soltera & $33(45.8)$ & $72(50.0)$ & $105(48.6)$ & \\
\hline Casada & $23(31.9)$ & $41(28.5)$ & $64(29.6)$ & \\
\hline Conviviente & $16(22.2)$ & $31(21.5)$ & $47(21.8)$ & \\
\hline Grado de Instrucción (\%) & & & & 0.016 \\
\hline Sin instrucción / Primaria & $14(19.4)$ & $10(6.9)$ & $24(11.1)$ & \\
\hline Secundaria & $44(61.4)$ & $109(75.7)$ & $153(70.8)$ & \\
\hline Superior & $14(19.4)$ & $25(17.4)$ & $39(18.1)$ & \\
\hline Distrito (\%) & & & & 0.646 \\
\hline Chorrillos & $10(13.9)$ & $14(9.7)$ & $24(11.1)$ & \\
\hline San Juan de Miraflores & $18(25.0)$ & $34(23.6)$ & $52(24.1)$ & \\
\hline Villa el Salvador & $15(20.8)$ & $33(22.9)$ & $48(22.2)$ & \\
\hline Villa María del Triunfo & $11(15.3)$ & $25(17.4)$ & $36(16.7)$ & \\
\hline Lurín/Pachacamac & $12(16.7)$ & $17(11.8)$ & $29(13.4)$ & \\
\hline Otros & $6(8.3)$ & $21(14.6)$ & $27(12.5)$ & \\
\hline
\end{tabular}

Del total de mujeres, los hallazgos más comunes del PAP fueron: Metaplasia escamosa y ASCUS. (Gráfico 2)

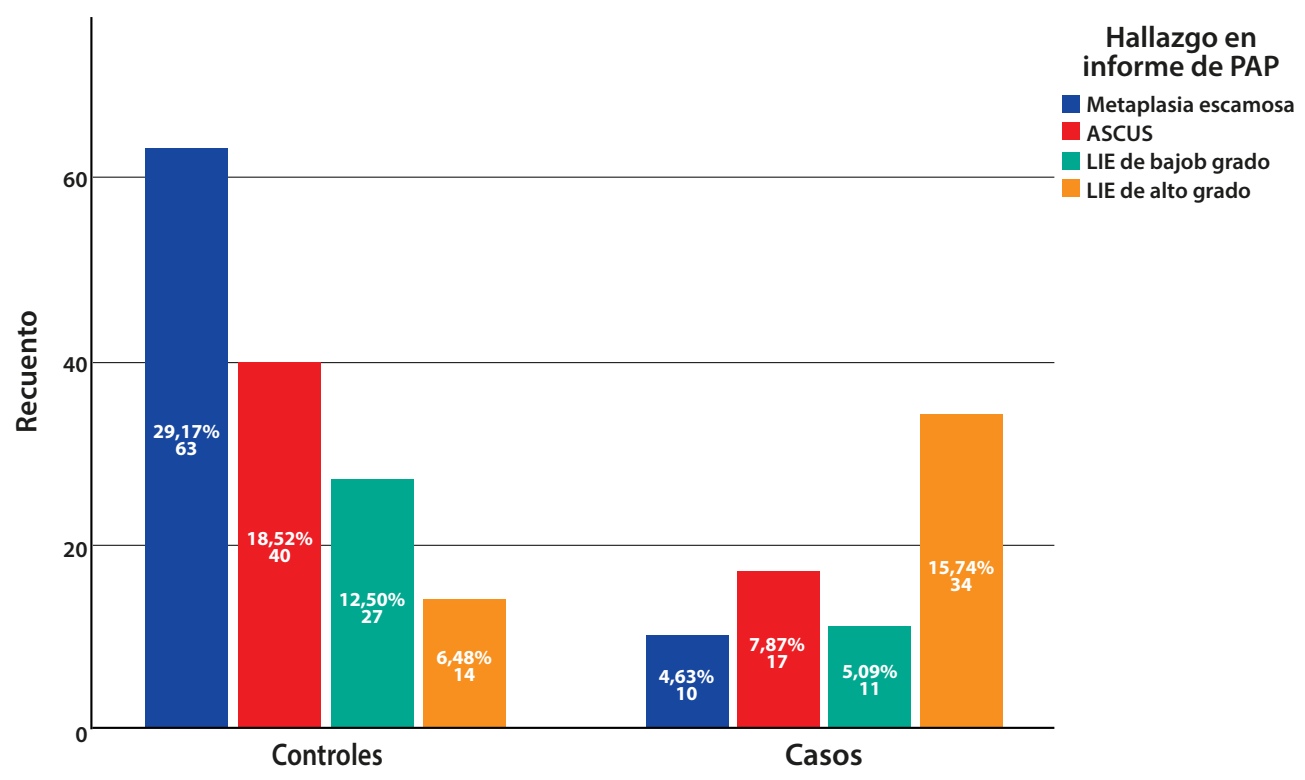

Fuente: Base de datos de las historias clínicas revisadas.

Gráfico 2. Distribución de hallazgos en informe de Papanicolau en mujeres con citología cervical anormal atendidas en el Hospital María Auxiliadora. 
Tabla 2. Características gineco-obstétricas de las pacientes con citología cervical anormal atendidas en el Hospital María Auxiliadora.

\begin{tabular}{|c|c|c|c|c|}
\hline & $\begin{array}{l}\text { CASOS } \\
(n=72)\end{array}$ & $\begin{array}{l}\text { CONTROLES } \\
(n=144)\end{array}$ & TOTAL & VALOR P \\
\hline Edad de menarquia (\%) & & & & 0.051 \\
\hline Menor de 11 & $24(33.3)$ & $28(19.4)$ & $52(24.1)$ & \\
\hline 11 a 14 años & $28(38.9)$ & $77(53.5)$ & $105(48.6)$ & \\
\hline Mayor a 14 años & $20(27.8)$ & $39(27.1)$ & $59(27.3)$ & \\
\hline Edad de inicio de relaciones sexuales (\%) & & & & 0.006 \\
\hline Menor de 15 años & $16(22.2)$ & $13(9.0)$ & $29(13.4)$ & \\
\hline 15 a 18 años & $41(56.9)$ & $78(54.2)$ & $119(55.1)$ & \\
\hline Mayor de 18 años & $15(20.8)$ & $53(36.8)$ & $68(31.5)$ & \\
\hline Múltiples parejas sexuales (\%) & & & & 0.001 \\
\hline SI & $51(70.8)$ & $64(44.4)$ & $113(52.3)$ & \\
\hline NO & $21(29.2)$ & $80(55.6)$ & $103(47.7)$ & \\
\hline Mayor a dos gestaciones (\%) & & & & 0.078 \\
\hline SI & $55(76.4)$ & $93(64.6)$ & $148(68.5)$ & \\
\hline NO & $17(23.6)$ & $51(35.4)$ & $68(31.5)$ & \\
\hline \multicolumn{5}{|l|}{ Mayor a dos partos (\%) } \\
\hline $\mathrm{SI}$ & $49(68.1)$ & $69(47.9)$ & $118(54.6)$ & \\
\hline NO & $23(31.9)$ & $75(52.1)$ & $98(45.4)$ & \\
\hline Método Anticonceptivo (\%) & & & & 0.390 \\
\hline Ninguno & $35(48.6)$ & $56(38.9)$ & $91(42.1)$ & \\
\hline Anticonceptivos orales / Inyectables & $27(37.5)$ & $63(43.8)$ & $90(41.7)$ & \\
\hline Otros (DIU, implante) & $10(13.9)$ & $25(17.4)$ & $35(16.2)$ & \\
\hline Hallazgos en PAP & & & & 0.000 \\
\hline Metaplasia escamosa & $10(13.9)$ & $63(33.8)$ & $73(43.8)$ & \\
\hline ASCUS & $17(23.6)$ & $40(27.8)$ & $57(26.4)$ & \\
\hline LIE de bajo grado & $11(15.3)$ & $27(18.8)$ & $38(17.6)$ & \\
\hline LIE de alto grado & $34(47.2)$ & $14(9.7)$ & $48(22.2)$ & \\
\hline Edad de diagnóstico & & & & 0.066 \\
\hline Menor de 25 años & $4(5.6)$ & $12(8.3)$ & $16(7.4)$ & \\
\hline 25 a 45 años & $39(54.2)$ & $96(66.7)$ & $135(62.5)$ & \\
\hline Mayor de 45 años & $29(40.3)$ & $36(25.0)$ & $65(30.1)$ & \\
\hline
\end{tabular}


Tabla 3. Análisis bivariado de los factores predisponentes para displasia cervical en las pacientes con citología cervical anormal atendidas en el Hospital María Auxiliadora.

$\begin{array}{lccc} & \text { OR } & \text { OROR } & \text { P } \\ \begin{array}{c}\text { Menarquia temprana } \\ \text { (SI/NO) }\end{array} & 2.071 & (1.09-3.93) & 0.024 \\ \begin{array}{c}\text { Inicio temprano de vida } \\ \text { sexual (SI/NO) }\end{array} & 2.213 & (1.14-4.29) & 0.017 \\ \begin{array}{c}\text { Múltiples parejas sexuales } \\ \text { (Mayor a 2/Menor a 2) }\end{array} & 3.036 & (1.66-5-56) & 0.001 \\ \begin{array}{c}\text { Múltiples gestaciones } \\ \text { (Mayor a 2/Menor a 2) }\end{array} & 1.774 & (0-93-3.37) & 0.078 \\ \quad & & & \\ \quad \text { Múltiples partos } \\ \text { (Mayor a 2/Menor a 2) }\end{array}$

Tabla 4. Análisis multivariado de los factores predisponentes para displasia cervical en las pacientes con citología cervical anormal atendidas en el Hospital María Auxiliadora.

\begin{tabular}{|c|c|c|c|}
\hline & OR & IC de $95 \%$ & $\mathbf{p}$ \\
\hline Menarquia temprana (SI/NO) & 1.507 & $(0.75-3.03)$ & 0.249 \\
\hline $\begin{array}{l}\text { Inicio temprano de vida } \\
\text { sexual (SI/NO) }\end{array}$ & 1.553 & $(0.76-3.17)$ & 0.227 \\
\hline $\begin{array}{l}\text { Múltiples parejas sexuales } \\
\text { (Mayor a 2/Menor a 2) }\end{array}$ & 2.626 & $(1.39-4.96)$ & 0.003 \\
\hline $\begin{array}{l}\text { Múltiples gestaciones } \\
\text { (Mayor a 2/Menor a 2) }\end{array}$ & 1.466 & $(0.23-2.01)$ & 0.488 \\
\hline $\begin{array}{c}\text { Múltiples partos } \\
\text { (Mayor a 2/Menor a 2) }\end{array}$ & 2.759 & $(1.20-7.46)$ & 0.045 \\
\hline $\begin{array}{c}\text { Uso de método } \\
\text { anticonceptivo (SI/NO) }\end{array}$ & 0.764 & $(0.41-1.42)$ & 0.395 \\
\hline
\end{tabular}




\section{DISCUSIÓN}

Diversos autores han mostrado evidencia de que un alto porcentaje de cánceres son causados por factores relacionados al estilo de vida, que en su mayoría, podemos cambiar. La displasia cervical es el cambio anormal en las células de la superficie del cuello uterino. El VPH es una causa importante pero no la única para desarrollar CCU; otros factores están involucrados en el proceso de carcinogénesis.

En el presente estudio, respecto a la edad de las pacientes estudiadas, el $63,9 \%$ se encontraba entre los 31 y 50 años de edad, dato que es congruente con investigaciones pasadas de Cifuentes (edad media: 42,2 años) ${ }^{12}$, Conde (edad de 25 a 35 años: $\left.72,9 \%\right)^{17}$ y Mesares (edad media de $39.87 \pm 10.08$ años) ${ }^{19}$.

Por otro lado, en el presente estudio la mayoría de las pacientes fueron solteras $(48,6 \%)$, mientras que en el trabajo realizado por Galván et al (México) el $81,7 \%$ de las pacientes eran casadas/unión libre ${ }^{13}$. Del mismo modo, en la tesis realizada por Aguilar (Perú), se obtuvo el estado civil conviviente como el más frecuente $(44,76 \%)^{18}$. Sin embargo, en la tesis de Enciso Cebrián (Perú) predominaron las pacientes solteras en un $36,8 \% .{ }^{26}$ Estos resultados diferentes nos indican que no existe asociación entre el estado civil y el riesgo de presentar displasia cervical, además que en nuestro estudio el valor de $\mathrm{P}=0.828$, lo que no es estadísticamente significativo.

Cabe mencionar que el nivel de instrucción fue el único ítem demográfico estadísticamente significativo, siendo el nivel secundario el más predominante $(70,8 \%)$, este resultado es similar al estudio de Mesares, donde el nivel secundario abarcó el $78,0 \%{ }^{19}$. No obstante, en otros trabajos como el de Aguilar y Ruiz, el grado de instrucción primario fue el más frecuente, en un $49,52 \%$ y $49,3 \%$ respectivamente ${ }^{18,15}$. Esto se relaciona con el nivel de conocimientos que tienen las pacientes respecto al cáncer cervicouterino, la infección por VPH y la vacuna contra este virus. Por ejemplo, en un estudio realizado por Valdéz en Andahuaylas (2015), el $66,7 \%$ de las participantes tenían un nivel de educación secundario, y de esa población el 37,5\% desconocían los beneficios de la vacunación contra el VPH, lo que significa que es necesario profundizar en la educación de las mujeres jóvenes sobre educación sexual y medidas preventivas contra el VPH a nivel naciona ${ }^{20}$.

Analizando los factores gineco-obstétricos, la edad de menarquia más frecuente (48,6\%) fue entre los 11 y 14 años de edad, hallazgo que se correlaciona con estudios como el de Galván ${ }^{13}$, Mesares ${ }^{19}$ y Cifuentes, ${ }^{12}$ donde la edad media de menarquia fue $12,7 \pm 1,50,12,88 \pm 1,49$ y

\section{$13,8 \pm 1,38$ años respectivamente.}

La edad de inicio de relaciones sexuales fue uno de los aspectos más estudiados en la mayoría de los trabajos, en el presente estudio el $55,1 \%$ de las mujeres estudiadas tuvo su primera relación sexual entre los 15 y 18 años, lo que coincide con los resultados obtenidos por Montesino et al, quienes hallaron que el $59,7 \%$ de las pacientes iniciaron su vida sexual antes de los 18 años, 11 así como el estudio de Montero, donde el 68,8\% de las participantes iniciaron su vida sexual entre los 15 y 19 años. 10 Del mismo modo, en nuestro estudio se encontró que el inicio temprano de relaciones sexuales elevaría el riesgo de presentar displasia cervical en 2,21 veces ( $P=0.017)$. Estos resultados son comparables a los obtenidos por Arotoma ( $\mathrm{OR}=2,757, \mathrm{P}=0.015), 16$ Galván $(\mathrm{OR}=2,5, \mathrm{P}=0.02) 13$ y Abarca $(\mathrm{OR}=1,95, \mathrm{P}=0.035)^{14}$, lo que significa que está altamente relacionado y es estadísticamente significativo.

Respecto al número de parejas sexuales, la condición de tener 3 a más parejas sexuales elevaría el riesgo para displasia cervical en 3,03 veces $(P=0.001)$, similar al resultado obtenido por Abarca et al, donde la misma condición presentó un $\mathrm{OR}=2,10(\mathrm{P}=0.0)^{14}$.

La multiparidad se presentó en el $54,6 \%$ de las pacientes estudiadas. En el presente estudio, el presentar 3 o más partos incrementaría el riesgo de esta enfermedad en 2,31 veces $(P=0.005)$. Este hallazgo coincide con los resultados obtenidos en los estudios de Conde $(\mathrm{OR}=3,835 ; \mathrm{P}=0.001)^{17}$ y Mesares $(\mathrm{OR}=2.364$; $\mathrm{P}=0.014)^{19}$, por lo que se acepta la multiparidad como factor estadísticamente significativos de riesgo ${ }^{28}$. Sin embargo, el presentar 3 o más gestaciones no fue estadísticamente significativo en la población estudiada ( $O R=1,774 ; P=0.078$ ). Similarmente, tampoco fue significativo en los estudios de Conde $(P=0.0543)^{17}$ y Galván $(P=0.376)^{13}$, por lo que esta condición no se considera como factor predisponente a presentar displasia cervical.

Por otro lado, el uso o no de métodos anticonceptivos se presentaría en este estudio como factor protector $(\mathrm{OR}=0.673)$, sin embargo, el valor de $\mathrm{P}$ no es estadísticamente significativo $(\mathrm{P}=0.173)$, por lo que no es comparable a otros estudios previamente mencionados, y en la población estudiada, se rechazaría como factor predisponente para displasia cervical. Así mismo, al hacer una correlación de Pearson entre el tipo de métodos anticonceptivos que usaban, sólo resultó positiva para las pacientes que no usaron métodos anticonceptivos. Sin embargo el uso del preservativo si se consideraría como un factor protector contra el VPH y la displasia cervical ya que es un método de barrera. 
Según el Centros de Control y Prevención de Enfermedades (CDC), en el calendario de vacunación para adultos, recomiendan la vacunación en mujeres hasta los 26 años, y a cualquier edad ${ }^{21}$. El programa de vacunación a nivel nacional incluye la vacuna contra el Virus del Papiloma Humano desde el año 2011, sin embargo, es a partir del 2016 que se realizan campañas para promover esta vacuna en niñas del 5 to grado de primaria de instituciones públicas y privadas $^{22}$. En un aplicativo web revisado en febrero 2019, donde se recuenta el número de niñas vacunadas gracias a la campaña nacional iniciada desde enero del 2017, se han vacunado 200,485 niñas, teniendo así una cobertura de $79.0 \%$ a nivel nacional y de 64,77\% en Lima Metropolitana23. Por esta razón es necesario continuar realizando estudios similares a este, para informar los resultados a las Redes de Salud a nivel nacional, y continuar las medidas de promoción y prevención del cáncer cervicouterino.

En un estudio de Mexico del 2018 señalan que existe la posibilidad de asociación entre el cáncer de cuello uterino ( $\mathrm{CaCu}$ ) y los cambios en el ecosistema vaginal a través de la modificación de la microbiota; y todas estas características del VPH y del huésped contribuyen a la inmunosupresión cervical y propician la carcinogénesis ${ }^{24}$. Otro estudio Mexicano del 2018 encontró predominio de comunidades bacterianas del género Fusobacterium. Al igual que otros estudios, también se encontró el predominio de Lactobacillus crispatus y Lactobacillus iners en los casos sin lesión en cérvix con infección positiva y negativa a VPH, respectivamente. Así mismo, el predominio de Sneathia spp en los casos con lesión premaligna en cérvix. Estos resultados del estudio del microbioma del cérvix en $\mathrm{CaCU}$ indican una diversidad y composición de la microbiota diferente en cada etapa de la historia natural del CaCU25 y estos avances en biología molecular permiten caracterizar y estimar la infección por VPH en diferentes poblaciones, localizaciones específicas y etiología.
Como se puede observar, a diferencia de otros cánceres, la mayoría de factores predisponentes son modificables, ya sea con cambios en los estilos de vida o con medidas preventivas como son la vacuna en las niñas y adolescentes, o el tamizaje de citología cervical en mujeres jóvenes. Además e intervalo para el progreso a cáncer invasor, típicamente puede ser mayor a 10 a 20 años. Así mismo, cerca de $70 \%$ de las lesiones ASCUS y NIC 1 desaparecen en un período de 6 años, mientras que alrededor de $6 \%$ de las lesiones NIC 1 progresan a NIC 3 o más. Entre 10 y $20 \%$ de las mujeres con lesiones $\mathrm{NIC} 3$, estas progresan a cáncer invasivo ${ }^{27-29}$.

Se recomienda desarrollar estudios a nivel molecular sobre el microbioma cervical como un modificador de la historia natural del virus del papiloma humano (VPH), con respecto al desarrollo de lesiones cervicales y neoplasias del cuello uterino.

\section{LIMITACIONES DEL ESTUDIO}

El estudio es unicéntrico, así mismo el número de casos es 72 lo que se compensa con los 144 controles. Este tipo de estudio solo nos permite detectar posibles asociaciones, y generar futuras hipótesis de relación causa efecto, en comparación con estudios multicéntricos, longitudinales y prospectivos.

\section{CONCLUSIÓN}

En el presente estudio se concluye que los factores del estilo de vida predisponentes a displasia cervical fueron tener múltiples parejas sexuales y la multiparidad.

Contribuciones de autoría: Los autores participaron en la concepción, recolección de información, redacción y aprobación de la versión final del artículo.

Financiamiento: Autofinanciado.

Conflicto de interés: Los autores declaran no tener conflicto de interés en la publicación de este artículo.

Recibido: 19 de noviembre del 2018

Aprobado: 20 de enero del 2019

Correspondencia: Rocío Gallegos Toribio

Dirección: Alameda Domingo Tristán y Moscoso 230 Conjunto Residencia "Los Próceres" Bloque Q-Dpto 22. Santiago de Surco, Lima-Perú Celular: +51945536679

Correo:rocio.gallegost@gmail.com 


\section{REFERENCIAS BIBLIOGRÁFICAS}

1.World Health Organization. Control integral del cáncer cervicouterino-Guía de prácticas [Internet]. World Health Organization. 2015.Disponible en:http:// iris.paho.org/xmlui/bitstream/handle/123456789/28512/9789275318799_ spa.pdf?ua $=1$

2. World Health Organization. Papilomavirus humanos (PVH) y cáncer cervicouterino [Internet]. World Health Organization. [citado 19 de junio de 2018]. Disponible en: http://www.who.int/es/news-room/fact-sheets/detail/ human-papillomavirus-(hpv)-and-cervical-cancer

3. GLOBOCAN. Cancer Fact Sheets: Cervical cancer [Internet]. GLOBOCAN [citado 19 de junio de 2018]. Disponible en: http://globocan.iarc.fr/old/ FactSheets/cancers/cervix-new.asp

4. Organización Mundial de la Salud. Perfiles oncológicos de los países, 2014. 2014

5. Ministerio de Salud. Analisis de la situacion del cancer en el Peru, 2013. Lima: Ministerio de Salud; 2013.

6. Coello Vásquez J. Compendio Estadístico 2015 [Internet]. Hospital María Auxiliadora: Oficina de estadística e informática; 2015 p. 131. Report No.: VII. Disponible en: http://www.hma.gob.pe/pdf/publicaciones/20.pdf

7. Walboomers JM, Jacobs MV, Manos MM, Bosch FX, Kummer JA, Shah KV et al, Human papillomavirus is a necessary cause of invasive cervical cancer worldwide. J Pathol 1999; 189(1): 12-9.

8. World Health Organization. Comprehensive cervical cancer control: a guide to essential practice - 2 ed, World Health Organization 2014

9. Kahn JA- HPV vaccination for the prevention of cervical intraepithelial neoplasia. N Engl J Med 2009; 361:271.

10. Montero Lora Y, Ramón Jimenez R, Valverde Ramón C, Escobedo Batista $F$, Hodelín Pozo E. Principales factores de riesgo en la aparición del cáncer cervicouterino. MEDISAN. 2018;5(1):8.

11. Montesino Aguiar JC, Arronte Santos ME, Matos Rodríguez A, Arias Puedmag $\mathrm{DE}$, Fernández $\mathrm{A}$. Comportamiento de factores de riesgo en pacientes con citologías anormales en el estado Miranda, Venezuela. Rev Cuba Obstet Ginecol. 2017;43(1):11

12. Cifuentes LY, Manrique FG, Ospina Díaz JM. Factores asociados al hallazgo de lesiones preneoplásicas detectadas en citología vaginal: estudio de caso y controles. Av En Enferm. 7 de octubre de 2014;32(1):63-71.

13. Galván Meléndez MF, Barragán Fernández $M$, Meléndez Hurtado RB. Factores de riesgo asociados a lesiones intraepiteliales escamosas de alto grado. 2013;(24):5.

14. Abarca Gómez L, Salas Estrada M, Calvo León D, Freer Vargas J, Cordero P. Factores asociados a las alteraciones del test de Papanicolaou en Costa Rica. 2014;5.

15. Ruiz Leud A, Bazán Ruiz S, Mejia CR. Hallazgos citológicos y factores de riesgo en citología cervical anormal en mujeres de pescadores del norte peruano, 2015. Rev Chil Obstet Ginecol. 2017;82(1):26-34.

16. Arotoma Oré M, Cayra Sahuanay O, Arotoma Nuñez E, Ríos Salinas R. Factores de riesgo para anormalidades citológicas del cuello uterino en pacientes atendidas en los establecimientos de salud, de la provincia de Huaraz, 2013. Aporte Santiaguino. 2015;8(1):10.
17. Conde Félix AM. Factores de riesgo asociados a lesiones en el cuello uterino en mujeres en edad fértil del Hospital María Auxiliadora Julio-Agosto 2013. [Hospital María Auxiliadora]: Universidad San Martín de Porres; 2014.

18. Aguilar Palomino GR. Factores de riesgo asociados a cáncer de cérvix en pacientes atendidas en el Hospital Vitarte durante el año 2015. [Lima] Universidad Ricardo Palma; 2017.

19. Mesares Salcedo E. Características personales y su asociación con los hallazgos de citología cervical de las usuarias que acudieron al Hospital San Juan de Lurigancho, enero - diciembre 2013. [Hospital San Juan de Lurigancho]: Universidad Nacional Mayor de San Marcos; 2015.

20. Valdez Castillo EG. Relación entre el nivel de conocimiento y las actitudes preventivas sobre la infección por VPH en usuarias del centro de salud "Chancas de Andahuaylas» de Santa Anita, Primer Semestre del 2015. Universidad Nacional Mayor de San Marcos; 2015.

21. Centers for Disease Control and Prevention 2018 Recommended Immunizations for Adult: By Health Condition [Internet]. Centers for Disease Control and Prevention. 2018. Disponible en: https://www.cdc.gov/vaccines/ schedules/downloads/adult/adult-schedule-easy-read.pdf

22. Ministerio de Salud (MINSA). Resolucion Ministerial N719-2018 [Internet] 2018. Disponible en: ftp://ftp2.minsa.gob.pe/normaslegales/2018/ Resolucion_Ministerial_719-2018-MINSA1.pdf

23. Ministerio de Salud (MINSA). Cobertura de Vacunación contra el VPH [Internet]. Vacunómetro VPH. 2019. Disponible en: http://appsalud.minsa. gob.pe/vacunometrovph/mapa/mapvacunaregion2.aspx

24. Vargas V. La asociación de la microbiota, virus del papiloma humano y cáncer cervicouterino. Departamento de Ginecología y Obstetricia de Hospital Juárez de México. Rev Hosp Jua Mex 2018; 85(1): 6-8. Disponible en: http://www.medigraphic.com/pdfs/juarez/ju-2018/ju181b.pdf

25. Madrid M., Torres K. La importancia de la microbiota cervicovaginal en cáncer cervicouterino. Facultad de Medicina; UNAM. Mens. Bioquim 42 (2018) 57-63. Disponible en: http://tab.facmed.unam.mx/files/6-MadridMarina.pdf

26. Enciso E. Factores de riesgo asociados a la displasia de cérvix en pacientes atendidas en la unidad de displasia del Hospital San José en el año 2016. Universidad Ricardo Palma. Facultad de Medicina Humana. Tesis. Disponible en: http://repositorio.urp.edu.pe/bitstream/handle/URP/1159/ TESIS\%20EDITH\%20PAMELA\%20ENCISO\%20CEBRI\%C3\%81 N\%2OHECHO. pdf? sequence $=1$ \&isAllowed $=y$

27. Holowaty $P$, Miller $A B$, Rohan $T$, et al:: Natural history of dysplasia of the uterine cervix. J Natl Cancer Inst 91 (3): 252-8, 1999. [PUBMED Abstract]

28. Melnikow J, Nuovo J, Willan AR, et al.: Natural history of cervical squamous intraepithelial lesions: a meta-analysis. Obstet Gynecol 92 (4 Pt 2): 727-35, 1998. [PUBMED Abstract]

29. Arends MJ, Buckley $\mathrm{CH}$, Wells M: Aetiology, pathogenesis, and pathology of cervical neoplasia. J Clin Pathol 51 (2): 96-103, 1998. [PUBMED Abstract]

30. Sellors J,Sankaranarayanan R. La colposcopia y el tratamiento de la neoplasia intraepitelial cervical: Manual para principiantes. Centro Internacional de Investigaciones sobre el Cáncer Lyon, Franc Disponible en: https://screening.iarc.fr/doc/colpoesmanual.pdf

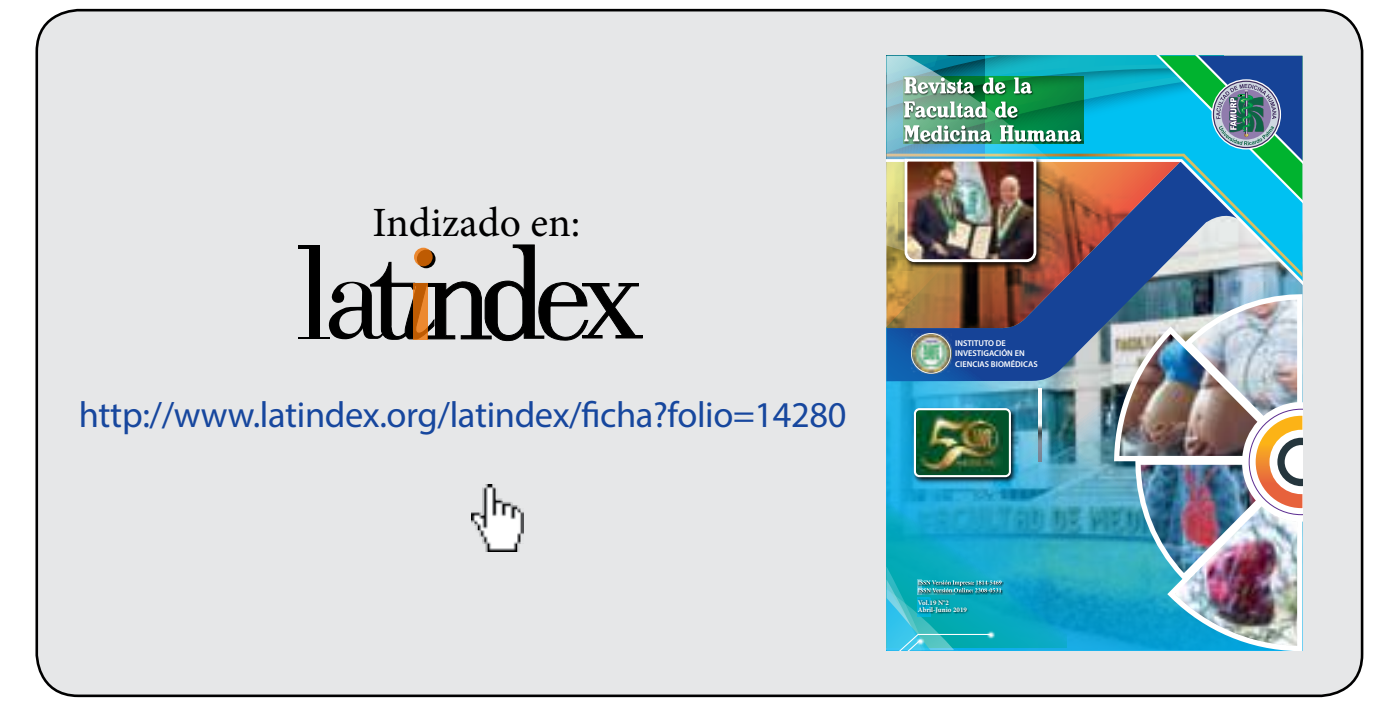

